\title{
Medium-Range Structure of Zr-Cu-Al Bulk Metallic Glasses from Structural Refinement Based on Fluctuation Microscopy
}

Jason J. Maldonis ${ }^{1}$, Pei Zhang ${ }^{1}$, Matt Besser ${ }^{2}$, Matt Kramer ${ }^{2}$, Paul M. Voyles ${ }^{1}$

${ }^{1}$ Department of Materials Science and Engineering, University of Wisconsin, Madison, WI, USA

${ }^{2}$ Ames Laboratory (DOE), Ames, Iowa, USA and Department of Materials Science and Engineering, Iowa State University, Ames, Iowa, USA

Bulk metallic glasses (BMGs) show a variety of outstanding properties including high yield strength [1], high elastic limit [2], and excellent nanoprocessability [3]. However, applications of BMGs are limited by the high cooling rates required to avoid crystallization and create a glass. Enhancing the glassforming ability (GFA) and understanding the origin of good GFA are therefore important to further applications of these alloys.

We have investigated the medium-range, nanometer-scale structure two $\mathrm{Zr}-\mathrm{Cu}$ - $\mathrm{Al}$ alloys with different GFA by combining experimental data from fluctuation electron microscopy (FEM) [4] with simulated system energies from an empirical interatomic potential in a hybrid reverse Monte Carlo (HRMC) structural refinement [4,5]. FEM data constrain the medium-range order of the structure, and the potential constrains the short-range and chemical order. HRMC optimizes the structure using the Metropolis algorithm and random, single-atom moves.

Fig. 1(a) shows FEM $V(k)$ data and simulated $V(k)$ from the HRMC structures for a $\mathrm{Zr}_{50} \mathrm{Cu}_{35} \mathrm{Al}_{15} \mathrm{BMG}$ in the as-quenched state and after annealing at $0.83 T_{g}$ for $10 \mathrm{~min}$ and $60 \mathrm{~min}$, compared to previous results for $\mathrm{Zr}_{50} \mathrm{Cu}_{45} \mathrm{Al}_{5}$, an alloy with higher GFA [4]. Because FEM is a diffraction technique, the structure it imposes on the HRMC models is well-visualized in reciprocal space. Fig. 2 shows the computed 3D reciprocal space for the model of the 60 minute, $300{ }^{\circ} \mathrm{C}$ annealed sample. Fig. 2(a) and (b) are isosurface images of the 3D reciprocal space, which identify volumes where significant diffraction occurs. Fig. 2(c) shows the most important atoms that contribute to the diffraction spots labeled in (a) and (b), identified by filtering and back transforming this pair of spots. This "sub-model" consists primarily of an ordered cluster of approximately 300 atoms near the center of the supercell along with some surrounding atoms. By rotating the sub-model with respect to the orientation of the vector $\boldsymbol{g}$, we can identify exactly which set of planes give rise to the diffraction from a specific pair of spots. This planar structure is shown (with planes vertical) in Fig. 2(d).

Models were also analyzed using radial distribution functions and Voronoi polyhedra (VP) indices [6]. VP indices were grouped into three categories: crystal-like, icosahedra-like, and mixed following Hwang et al. [4]. Analysis of the "sub-model" in Fig. 2(c) enables us to connect the feature in $V(k)$ at the $k=|g|=0.42 \AA^{-1}$ from Fig. 2(a), to crystal-like structure in the model. Fig. 1(b) shows that the fraction of crystal-like VP increases as a function of annealing, while the number of icosahedral-like VP simultaneously decreases. This indicates that the planar structure in $\mathrm{Zr}_{50} \mathrm{Cu}_{35} \mathrm{Al}_{15}$ becomes more crystallike with annealing at the cost of icosahedral-like local structures, which is opposite to the trend found for the better glass former, $\mathrm{Zr}_{50} \mathrm{Cu}_{45} \mathrm{Al}_{5}$ [4]. For the poorer glass former, the crystal-like peak has larger $V(k)$ while the icosahedral-like peak is smaller. Overall, results suggest that achieving better glass forming ability in this alloy system may depend on destabilizing crystal-like structures as much as enhancing non-crystalline ones. 


\section{References}

[1] C. Hays et al, Phys. Rev. Lett. 84 (2000), p. 2901.

[2] R. Vaidyanathan et al, Acta Mater. 49 (2001), p. 3781.

[3] M. Carmo et al, ACS Nano 5 (2011), p. 2979.

[4] J. Hwang et al, Phys. Rev. Lett. 108 (2012), p. 195505.

[5] M. M. J. Treacy and K. B. Borisenko, Science 335 (2012), p. 950.

[6] S. Hao et al, Phys. Rev. B 79 (2009), p. 104206.

[7] This work was supported by the NSF (DMR-1205899 and CMMI-1232731, JJM, PZ, and PMV). The facilities and instrumentation for microscopy were supported by the University of Wisconsin Materials Research Science and Engineering Center (DMR-1121288). The synthesis of alloys was supported by U.S. DOE, Office of Basic Energy Sciences, through the Ames Laboratory (MFB and MJK), Iowa State University under contract DE-AC02-07CH11358.
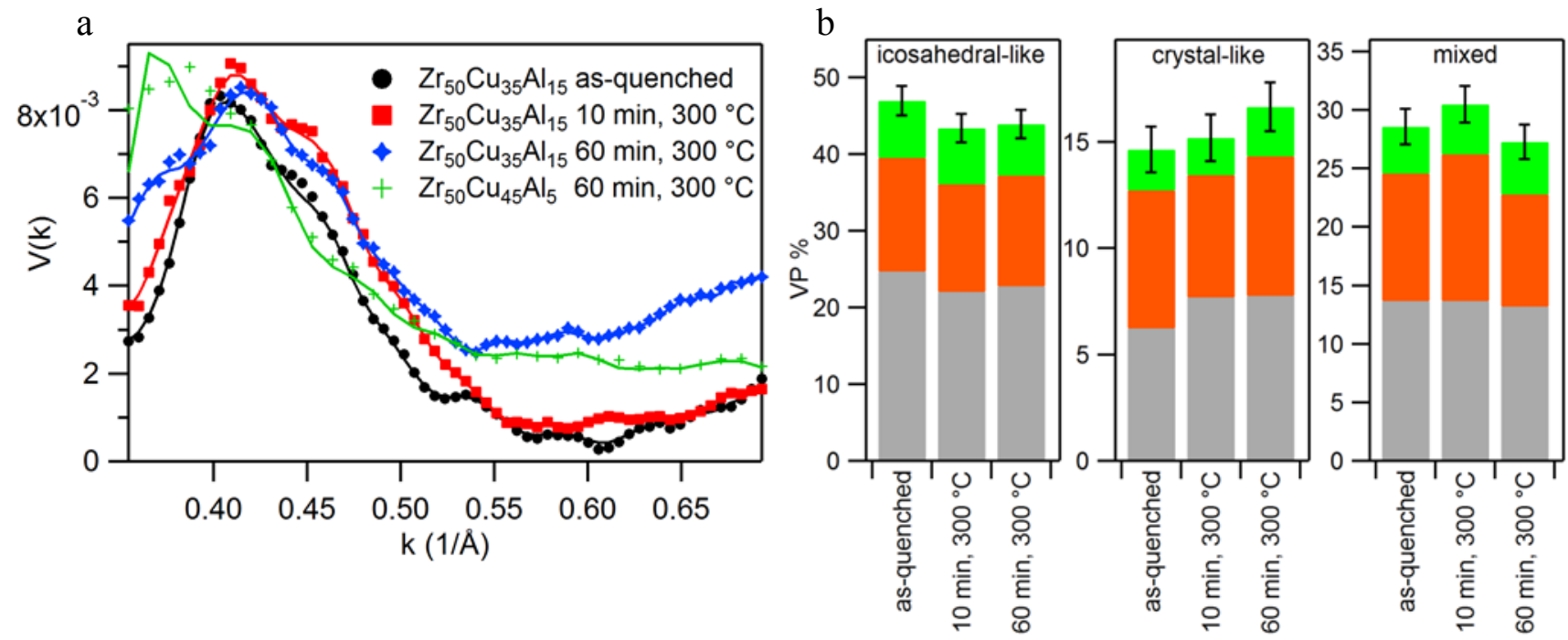

Figure 1. a) Experimental $V(k)$ (markers) and HRMC simulated $V(k)$ (lines) for two MGs. b) Histograms of VP categories for the $\mathrm{Zr}_{50} \mathrm{Cu}_{35} \mathrm{Al}_{15} \mathrm{MG} \mathrm{HRMC}$ models. From top to bottom: Al-centered (green), Cu-centered (orange), Zr-centered (gray) VPs.
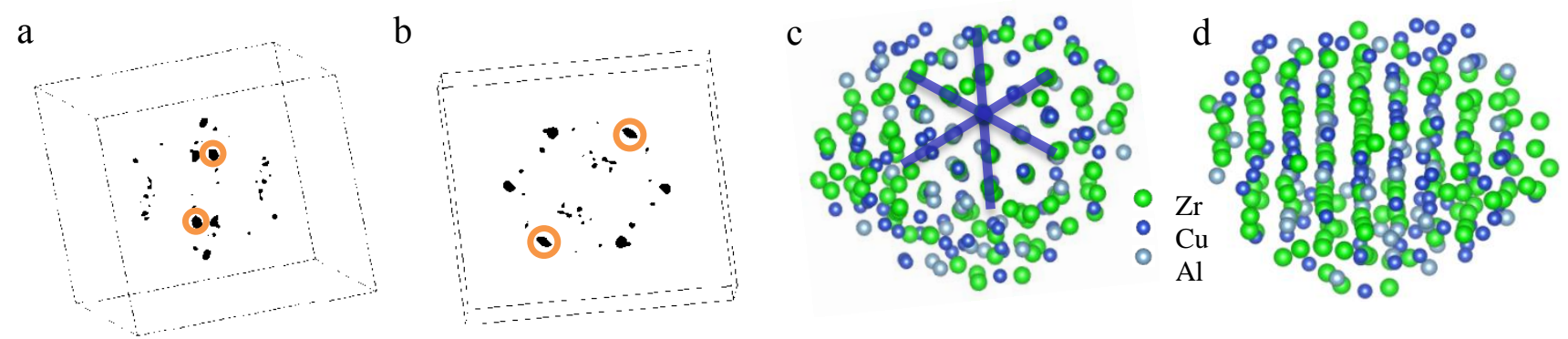

Figure 2. a-b) Two orientations of an isosurface plot of the $3 \mathrm{D}$ reciprocal space for $\mathrm{Zr}_{50} \mathrm{Cu}_{35} \mathrm{Al}_{15}$. c) The sub-model that results from the circled spots in (a) and (b). d) The sub-model in (c) reoriented corresponding to the direction of $g$. 